\title{
Redox Activity of an Organosulfur Compound and Its Application to Polymer Lithium Batteries
}

\author{
Osamu HATOZAKI and Noboru OYAMA*
}

\author{
Department of Applied Chemistry, Faculty of Technology, Tokyo University of Agriculture and Technology \\ (2-24-16 Naka-cho, Koganei, Tokyo 184-8588, Japan)
}

Received August 10, 1999 ; Accepted August 27, 1999

\begin{abstract}
Redox activity of an organosulfur compound with multiple thiol groups and its application to a cathode active material of lithium batteries are described. To accelerate a slow redox reaction of organosulfur compounds such as 2,5-dimercapto-1,3,4-thiadiazole (DMcT), conducting polymer polyaniline (PAn) is mixed with organosulfur compounds, yielding a composite material. Within the composite, DMcT and PAn interact at molecular-level and the redox reaction of DMcT is successfully accelerated by PAn. The DMcT/PAn composite shows a promise as a high energy cathode active material with the discharge capacity of $185 \mathrm{Ah} / \mathrm{kg}$-cathode and the average discharge voltage at $3.4 \mathrm{~V}$. Furthermore, using a copper foil as a cathode current collector, battery performances of the composite cathode are significantly improved. The observed improvement is attributed to a formation of a complex between a copper ion and DMcT, which accelerates the redox reaction of DMcT. The discharge capacity of the composite cathode on a copper current collector is $225 \mathrm{Ah} / \mathrm{kg}$-cathode. The discharge capacity is further increased to $500 \mathrm{Ah} / \mathrm{kg}$-cathode by adding elemental sulfur to the composite.
\end{abstract}

\section{Key Words : Organosulfur Compounds, Polyaniline, Composite Cathodes, Polymer Lithium Batteries}

\section{Introduction}

In the progress of modern technology, rechargeable batteries with high charge-discharge performances have increasingly become of importance for electroenergy storage and supply. Lithium batteries, which are characterized by their extremely high energy density due to high charge capacity and high output voltage, are currently in widespread use as a power source for small portable electronic devices such as cellular phones, camcorders, etc. Recently, use of lithium batteries as a power source for electric vehicles and load leveling in electric power is examined and thus lithium batteries with higher energy density are in great demand. The high energy density obtainable with lithium batteries is largely due to the charge density of the anode active materials such as lithium metal $(3861 \mathrm{Ah} / \mathrm{kg})$ and lithium intercalation carbon materials $(372 \mathrm{Ah} / \mathrm{kg}$ ). Therefore, development of stable cathode materials possessing higher charge density than $137 \mathrm{Ah} / \mathrm{kg}$ of lithium cobalt oxide $\left(\mathrm{LiCoO}_{2}\right)$, which is currently employed in a commercially available lithium-ion battery, would be efficient to increase the energy density to satisfy various demands which existing batteries barely meet.

Various inorganic and organic compounds are now examined as the cathode active materials for new lithium batteries. A series of organosulfur compounds with multiple thiol groups (-SH) is expected to be one of good candidates as the cathode material. The function of these compounds as an electrode active material is based on the reversible reaction of $2-\mathrm{SH} \rightleftarrows-$
S-S-. Organosulfur compounds can store a charge per thiol group and thus the theoretical capacity of, for example, 2,5-dimercapto-1,3,4-thiadiazole, DMcT, which has two thiol groups, is $362 \mathrm{Ah} / \mathrm{kg}$, far exceeding the capacity of $\mathrm{LiCoO}_{2}$. Compared to inorganic active materials used in a commercially available rechargeable battery, organosulfur compounds are environmentally benign.

As described just above, organosulfur compounds possess several characteristics which are very attractive as a cathode material for lithium batteries. However, the kinetics of the redox reaction of organosulfur compounds are rather slow at room temperature, which severely retards the use of these compounds for practical batteries. Recently, the authors have found that conducting polymer polyaniline (PAn) shows electrocatalytic activity towards both of the oxidation and reduction of organosulfur compounds, and proposed a composite material composed of PAn and organosulfur compounds as a novel cathode active material for lithium batteries operating at ambient temperature., ${ }^{1,2)}$ In the composite, PAn also acts as a molecular current collector for the insulating organosulfur compounds as well as an electrode active material. Furthermore, when a copper foil was used as a cathode current collector, it is observed that charge-discharge performances are significantly improved.

In this article, the redox characteristics of organosulfur compounds (DMcT), charge-discharge characteristics of a DMcT/PAn composite cathode material and the effect of a copper current collector 


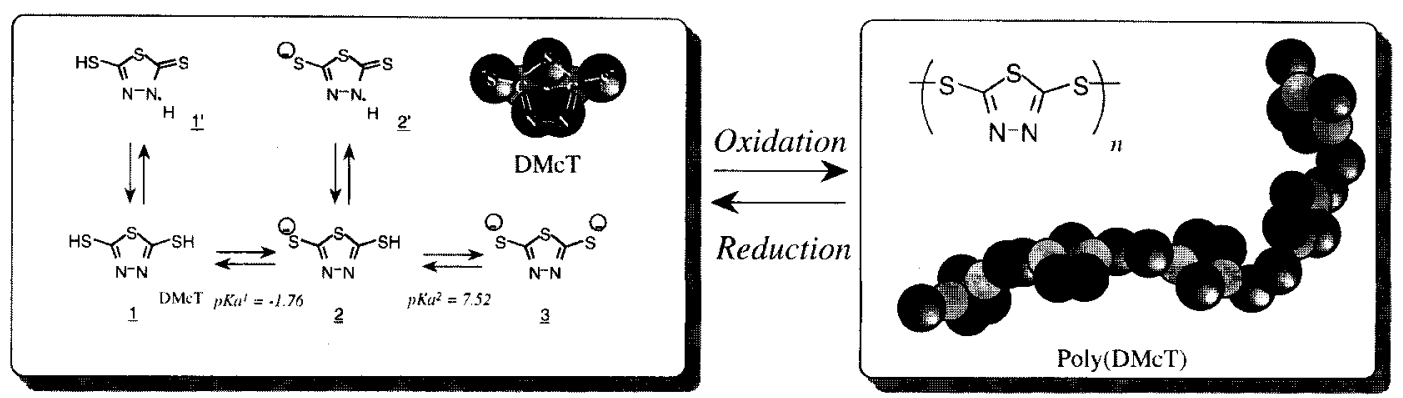

Scheme 1 Polymerization and depolymerization reactions of the disulfide bond in the redox reactions of DMcT.

are described based on our research results. The future prospects of our organosulfur-based lithium batteries are also discussed.

\section{Redox Activity of Organosulfur Compounds}

The reversible conversion of an organosulfur compound such as DMcT between polymerized and depolymerized forms corresponds to the oxidation of thiol groups and the following formation of the disulfide bond and the reduction and cleavage of the disulfide bond, respectively, as shown in Scheme 1. Because there exist proton dissociation equilibria for the thiol groups on organosulfur compounds (Scheme 1), ${ }^{3,4)}$ the redox activities of these compounds are strongly dependent on the acidity of a medium. ${ }^{4-7)}$ Figure 1 clearly shows the acidity dependent redox activity of DMcT. DMcT is oxidized and the disulfide bond was reduced at more positive potentials in an acidic medium (Fig. 1A) compared with those in a

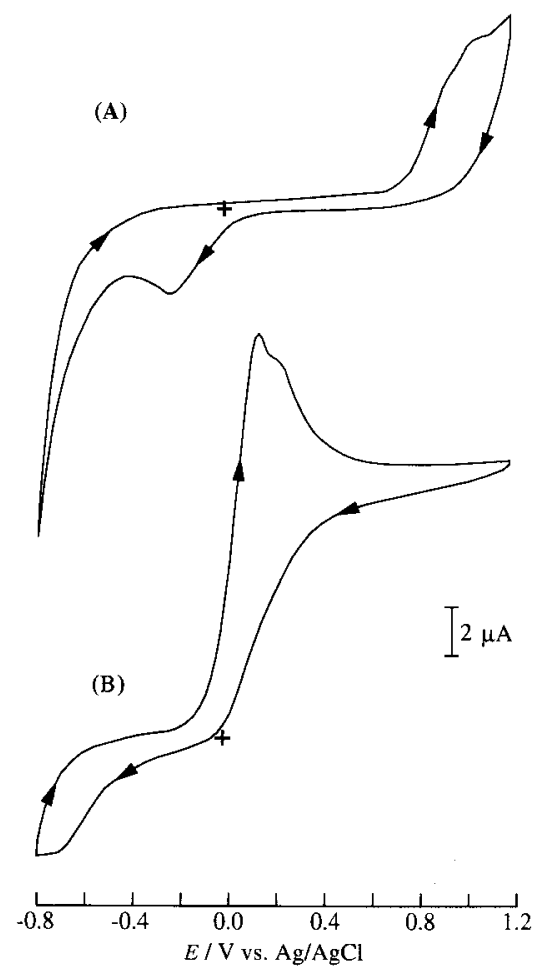

Fig. 1 Cyclic voltammograms of $10 \mathrm{mM} \mathrm{DMc} \mathrm{T}$ in the presence of (A) $25 \mathrm{mM}$ methansulfonic acid and (B) $25 \mathrm{mM}$ triethylamine in acetonitrile solutions containing $0.1 \mathrm{M}$ $\mathrm{LiClO}_{4}$. Electrode : glassy carbon. Scan rate : $50 \mathrm{mV} / \mathrm{s}$. basic medium (Fig. 1B). Based on $p K a$ values of the thiols on DMcT ${ }^{4)}$ in the presence of methansulfonic acid in excess of twice of DMcT, all of the thiol groups on DMcT monomer remains undissociated, while in the presence of excess triethylamine all of the thiols turn to be the proton-dissociated thiolate forms $\left(-\mathrm{S}^{-}\right){ }^{8)}$ Therefore, it is indicated that upon the proton dissociation of the thiols, the oxidation of DMcT becomes easier and the reduction of disulfide bonds in DMcT oxidation products becomes difficult.

Semiempirical molecular orbital (MO) calculations were carried for the DMcT monomers and dimers which were expected to give the redox reactions shown in Fig. 1. ${ }^{4}$ The MO calculations of the DMcT monomers and dimers indicated that the proton dissociation of the thiols on DMcT increased the energy of the HOMO level which localized on the sulfur atom of the thiol group, indicating that the oxidation of the thiolate is easier than that of the thiol. On the contrary, it was found that the proton dissociation increased the energy level of LUMO of the dimers which localized on the disulfide bond and had an antibonding nature, meaning that the reduction of the disulfide bond in the oxidized form becomes difficult upon the proton dissociation of the thiols. Additionally the MO calculation results suggested that oxidation and reduction activities of DMcT oligomers (higher oxidation products than a dimer) were not significantly influenced by change in chain length.

\section{Application of Organosulfur/PAn Compos- ite Cathode to Lithium Rechargeable Batteries with High Energy Density}

\section{1 Electrocatalysis of PAn towards the redox} reactions of organosulfurs

Organosulfur compounds are very attractive as an electrode active material because of their high theoretical gravimetric capacity. Visco et al. have shown the promise of organosulfur (RSSR) compounds as a cathode active material with high energy density. ${ }^{9-12)}$ For example, they reported that an organosulfurbased cathode with a lithium metal anode gave an energy density of $160 \mathrm{Wh} / \mathrm{kg}$ with moderate cyclability (over 350 charge-discharge cycles). ${ }^{9}$ However, these reasonable battery performances were achieved at only elevated temperatures (50-93 ${ }^{\circ}$ ), because of slow redox reactions of organosulfur com- 
pounds. ${ }^{13)}$ Therefore, to utilize the high charge density of organosulfur compounds at room temperature, the slow redox reactions had to be somehow accelerated. Furthermore, use of pure organosulfur compounds as a cathode active material would be hampered by the lack of electronic conductivity. Recently, the authors have successfully solved these problems by making a composite with conducting polymer polyaniline (PAn). PAn accelerates the slow redox reactions of organosulfur compounds and imparts electronic conductivity to organosulfur cathode materials. ${ }^{1)}$ Furthermore, PAn itself acts as a cathode active material. ${ }^{14}$

It is often suggested that molecular-level interactions among components are of importance to derive new useful properties as a composite. ${ }^{15)}$ Therefore, to increase the intimate interaction between DMcT and PAn, DMcT/PAn composites were prepared as follows: DMcT was dissolved in NMP and then PAn was added to the $\mathrm{DMcT} / \mathrm{NMP}$ solution with vigorous stirring. The obtained viscous black inky solution was spread on an electrode surface and dried under vacuum at $80{ }^{\circ} \mathrm{C}$ for $1 \mathrm{hr}$. While preparing the composite solution, it was found that solubility of PAn in NMP was higher in the presence of DMcT, which implied presence of chemical and/or physical interaction(s) between DMcT and PAn. We expect that this preparation method allows intimate molecular-level mixing of DMcT with PAn.

The electrocatalytic acceleration of the redox reactions of organosulfur compounds by PAn is seen from the enhancement of the redox current of PAn in the presence of DMcT as shown in Fig. 2. The redox current of a DMcT/PAn composite film coated on a gold electrode (Fig. 2A) is larger than that of a PAn film without DMcT (2B), suggesting that DMcT is incorporated into the PAn film and oxidized and

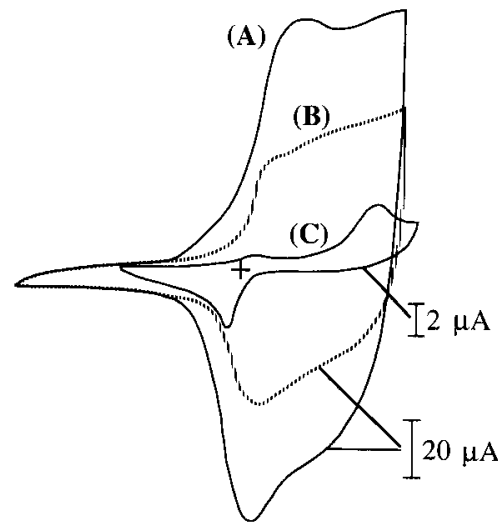

$$
\begin{array}{cccc} 
& & 1 & \perp \\
-0.8 & -0.5 & 0.0 & 0.5 \\
& E / V \text { vs. } \mathrm{Ag} / \mathrm{AgCl} &
\end{array}
$$

Fig. 2 Cyclic voltammograms of (A) the DMcT/PAn composite film coated on a gold electrode and (B) PAn film coated on a gold electrode. (C) Cyclic voltammogram of DMcT at a bare gold electrode. All voltammograms

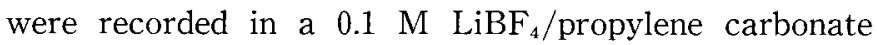
solution at a scan rate of $50 \mathrm{mV} / \mathrm{s}$. reduced via PAn in a potential range where PAn shows its redox activity. Compared with a cyclic voltammogram of DMcT at a bare gold electrode (2C), reversibility of the DMcT redox reaction is significantly improved by virtue of the electrocatalytic activity of PAn. Note that PAn accelerates both of the oxidation and reduction of organosulfur compounds.

The electrocatalytic activity of PAn for the DMcT redox reaction depends on the kinetics of electrontransfers between them. It was found that the rate of the electron-transfer from DMcT monomer to PAn was significantly augmented by the proton dissociation from the thiol groups and, especially, protonation of PAn. Therefore, acidity control in the composite will be required for the optimization of reactions within the composite.

\subsection{Characteristics of organosulfur/PAn compos- ite cathodes}

Charge-discharge characteristics of the DMcT/PAn composite was examined with a test cell using a lithium metal anode and a polymer gel electrolyte. ${ }^{1)}$ Discharge capacity of the cell increased with increas. ing in charging voltage. When a test cell was charged at $4.75 \mathrm{~V}$, the composite cathode gave discharge capacity of over $185 \mathrm{Ah} / \mathrm{kg}$-cathode, while at $4.0 \mathrm{~V}$ charging voltage the capacity was $c a .100 \mathrm{Ah} / \mathrm{kg}$ cathode. The capacity value of $185 \mathrm{Ah} / \mathrm{kg}$-cathode corresponded to more than $80 \%$ of the theoretical capacity of $224 \mathrm{Ah} / \mathrm{kg}$-cathode. This indicates that both DMcT and PAn successfully act as a cathode active material. When a test cell was charged at 4.75 $\mathrm{V}$, average output voltage was observed to be $c a .3 .4$ $\mathrm{V}$, which gave gravimetric energy density exceeding $600 \mathrm{Wh} / \mathrm{kg}$-cathode.

Although the DMcT/PAn composite cathodes exhibited high energy density, charging and discharging was still limited to too slow rates for practical use. The addition of a polypyrrole derivative enabled charging at a higher rate without undue deterioration of energy density. That is, the polypyrrole derivative functioned as a subsidiary molecular current collector.

It is worth noting that the composite formation offered another benefit of maintenance of electroactivity and thus electrocatalytic activity of PAn. Generally, PAn loses its electroactivity due to the loss of proton in overoxidation at a higher potential. ${ }^{16)}$ However, inside the composite, DMcT acts as a proton source for PAn and as a result stability of PAn is significantly improved even at $4.75 \mathrm{~V}$ vs. $\mathrm{Li} / \mathrm{Li}^{+}$.

\section{Effects of a Copper Current Collector on a Battery Performance of DMcT/PAn Composite Cathodes}

Battery performances of the DMcT/PAn composite cathode were investigated with various current collectors including copper, nickel, aluminum, titanium, gold on a carbon, and porous carbon. A test cell using a copper current collector for the composite cathode 
exhibited better cycle performances at higher charge and discharge rates compared with cells with the other current collectors. ${ }^{17)}$ Figure 3 shows results of charge-discharge testing of test cells with a copper and carbon cathode current collectors. The cells were charged and discharged at $0.83 \mathrm{C}(1 \mathrm{C}$ corresponds to a charge or discharge rate at which all of active materials are charged or discharged in one hour). A test cell with a copper current collector gave a quite stable cell performance. Discharge capacity of the cell was about $150 \mathrm{Ah} / \mathrm{kg}$-cathode. When the cell was charged and discharged at $0.5 \mathrm{C}$, discharge capacity increased to $225 \mathrm{Ah} / \mathrm{kg}$-cathode (estimated from the amount of DMcT and PAn contained in the cathode) with excellent cyclability over 250 charge-discharge cycles. Because the value of the capacity exceeded the theoretical capacity of $186 \mathrm{Ah} / \mathrm{kg}$-cathode, it was concluded that the copper current collector also functioned as an active material at the interface between the composite and the current collector (and within the composite).

Similar improvements of the battery performance were observed whencopper salts were added to the composite. ${ }^{18)}$ Since it is known that DMcT forms a stable complex with copper ions, ${ }^{19,20)}$ the observed improvements of the battery performance of the com posite cathode were expected to be associated with a formation of a complex between copper ion and $\mathrm{DMcT}$. To see about the formation of DMcT-Cu complex, surface observation was carried out for a copper substrate soaked in a solution containing $\mathrm{DMcT}$. Spontaneous dissolution of a copper substrate was observed in the presence of DMcT using electrochemical quartz crystal microbalance (EQCM) and phase measurement interferometric microscopy (PMIM). ${ }^{21,22)}$ The formation of a complex was confirmed by X-ray photoelectron spectroscopy (XPS), which showed peaks corresponding to $-\mathrm{Cu}-\mathrm{N}$ - and -Cu-S-. XPS studies indicated the presence of a complex layer at the interface between the copper current collector and the composite film. ${ }^{22}$ Cyclic voltammetric studies confirmed electrocatalytic activity of a $\mathrm{Cu}(\mathrm{I})-\mathrm{DMcT}$ complex towards the redox reactions of DMcT. Furthermore, from XPS spectra of the com-

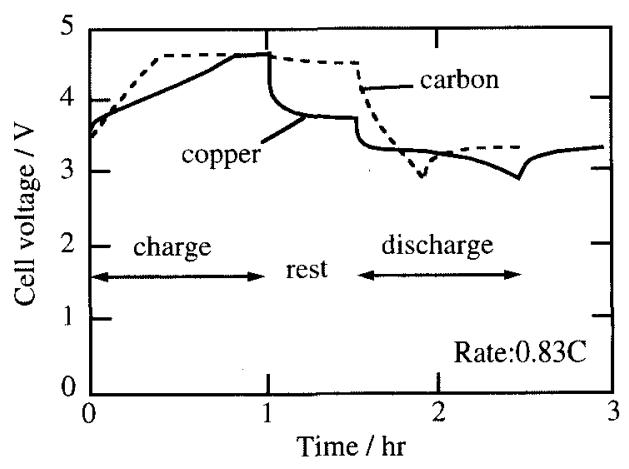

Fig. 3 Charge/discharge curves of test cells with (-) a copper and (--) carbon cathode current collectors. The cells were charged and discharged at a rate of $0.83 \mathrm{C}$. plex obtained at different electrode potentials, it was suggested that the electrocatalytic activity was based on the redox reaction of the $\mathrm{Cu}$ center of the complex. ${ }^{23)}$

\section{Future Prospects}

Elemental sulfur, $\mathrm{S}_{8}$, is also attractive as a cathode active material with high energy density. The theoretical capacity of $S_{8}$ is calculated to be $1675 \mathrm{Ah} / \mathrm{kg}$, assuming the number of electrons transferred per sulfur atom to be $n=2\left(\mathrm{~S}_{8} \rightleftarrows 8 \mathrm{~S}^{2-}\right)$. However, cyclic voltammetric responses of $S_{8}$ in a nonaqueous solution such as NMP show that $n$ is much less than 2 and output potential is expected to be lower than $3 \mathrm{~V}$, that is, practical energy density of $S_{8}$ being much lower than expected. Recently we prepared a composite cathode material containing $S_{8}$ by simply adding $S_{8}$ to NMP solutions dissolved with DMcT and PAn. Figure 4 shows discharge capacity of a test cell with $\mathrm{DMcT} / \mathrm{PAn} / \mathrm{S}_{8}$ composite cathode. Extremely high discharge capacity exceeding $500 \mathrm{Ah} / \mathrm{kg}$-cathode was attained with this composite cathode containing $\mathrm{S}_{8 .}{ }^{24}$ This value suggests that at least $70 \%$ of the sulfur in the composite was reduced to $\mathrm{S}^{-}$oxidation state during discharging process. Furthermore, average output voltage of the cell was $3.5 \mathrm{~V}$, which was much higher than expected from reported redox potentials of $\mathrm{S}_{8}$. These observations imply that $\mathrm{S}_{8}$ interacts with $\mathrm{DMcT}$ and/or PAn to yield an adduct which induced the observed battery performances. We expect that composite cathode material containing $\mathrm{S}_{8}$ is a good candidate for a cheap cathode active material with high energy density. Research on the composite containing $S_{8}$ is now under way to understand reactions within the composite in detail.

Recently, extensive studies are carried out for development of a polymer lithium battery which utilizes a polymer material as a cathode material and/or electrolyte (Fig. 5). The use of polymer materials as main components of a battery offers some advantages. For example, a polymer lithium battery can be a lightweight, thin battery with high flexibility. Furthermore, it is expected that the use of a polymer

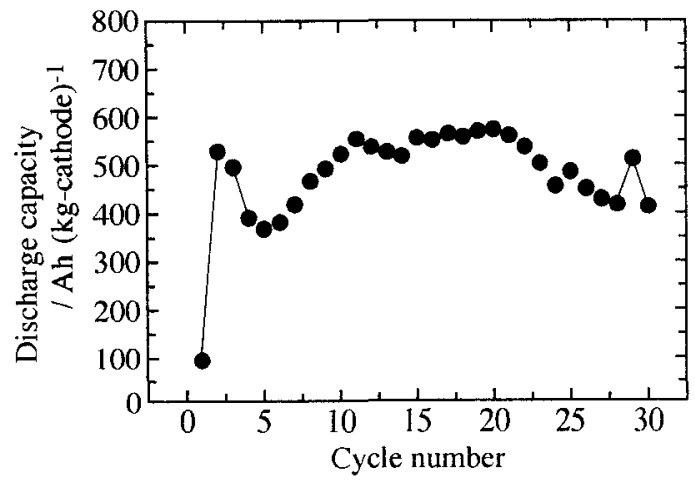

Fig. 4 Discharge capacity of a metal Li anode/ (PC/ $\left.\mathrm{EC}(1: 1)-\mathrm{LiBF}_{4}\right) / \mathrm{AN}-\mathrm{VAc}$ gel $) /\left(\mathrm{S}+\mathrm{DMcTLi}_{2}+\mathrm{PAn}\right)$ test cell at $1 \mathrm{~mA}$ at $20{ }^{\circ} \mathrm{C}$. PC: propylene carbonate, EC: ethylene carbonate, AN-VAc: acrylonitrile-vinylacetate copolymer. 
gel electrolyte in place of liquid electrolyte would allow the use of lithium metal as an anode material, because polymer gel electrolytes are reported to suppress the formation of lithium dendrites at the interface between $\mathrm{Li}$ and electrolyte, even at a high current density of $3 \mathrm{~mA} / \mathrm{cm}^{2}$ for $1 \mathrm{hr}{ }^{25)}$ Combining a DMcT/ PAn composite cathode with Li metal anode and gel electrolytes with high ionic conductivity, it is expected that polymer lithium batteries with unprecedentedly high energy density would be achieved. Furthermore, a unit cell of polymer batteries can be stacked into the layerd-structure and thus higer output voltage would be attained. Polymer batteries can be packaged with polyethylene-coated aluminum film instead of a steal container used for a lithium ion battery (Fig. 6). Those polymer lithium batteries would be useful as a power source for not only portable electronic devices but also for electric vehicles, robots and space crafts and stations, which demand

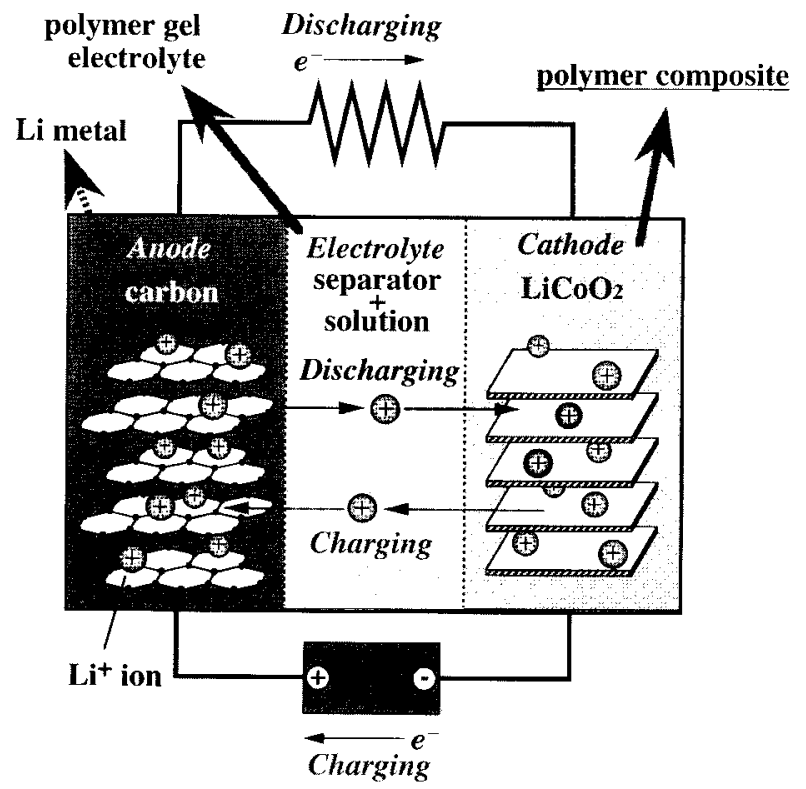

Fig. 5 Schematic depiction of the structure and chargetransfers of a lithium-ion battery. Polymer lithium batteries will use various polymer materials in place of a metal oxide cathode active material and liquid electrolyte.

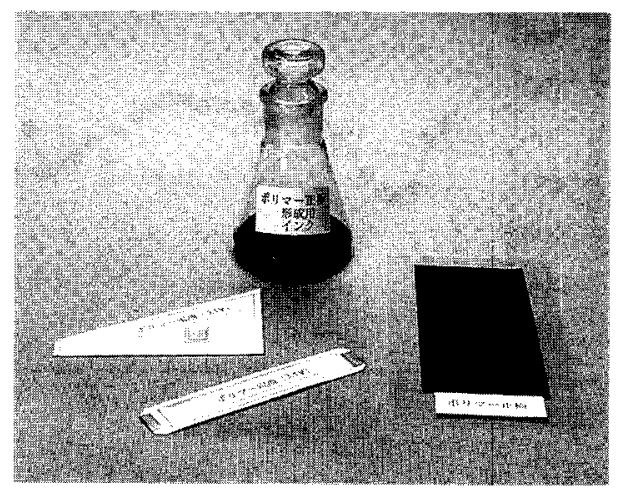

Fig. 6 Inky solution dissolving $\mathrm{DM}_{\mathrm{C}} \mathrm{T}$ and $\mathrm{PAn}$ in $\gamma$ butyroiactone and a thin, flexible polymer lithium battery with DMcT/PAn composite cathode packaged with a polyethylene-coated aluminum film. batteries to be accommodated into an empty space.

\section{Summary}

A composite material composed of organosulfur compounds and polyaniline (PAn) is highly promising as a cathode active material for lithium batteries. Test cells of polymer lithium battery using the composite cathode with polymer gel electrolyte and lithium metal anode exhibited high energy density and excellent cyclability. It was often observed that battery performance (e.g. cyclability) of these test was limited by the lithium metal anode. Charge-transfer reactions within the composite cathode are strongly affected by the acidity in the composite. Furthermore, using a copper substrate as a cathode current collector, the composite cathode could be charged and discharged at a practical rate. The obtained improvements of the battery performance due to a copper current collector was attributed to the formation of a $\mathrm{Cu}(\mathrm{I})$-DMcT complex which further accelerated the redox reaction of DMc T as shown in Scheme 2.

\section{Acknowledgment}

The authors are grateful to Drs. T. Tatsuma, J. M. Pope, F. Matsumoto, E. Shouji, Q. Chi, S. C. Paulson, M. Iwaku and T. Sotomura. This study was supported in part by the Proposal-Based New Industry Creative Type Technology R\&D Promotion Program from the New Energy and Industrial Technology Development Organization (NEDO) of Japan.

\section{References}

1) N. Oyama, T. Tatsuma, T. Sato, and T. Sotomura, Nature, 373, 598 (1995).

2) T. Sotomura, H. Uemachi, Y. Miyamoto, A. Kaminaga, and N. Oyama, Denki Kagaku(presently Electrochemistry), 61, 1366 (1993).

3) B. Stanovnik and M. Tisler, Croat. Chem. Acta., 37, 17 (1965).

4) E. Shouji, H. Matsui, and N. Oyama, J. Electroanal. Chem., 417, 17 (1996).

5) E. Shouji and N. Oyama, J. Electroanal. Chem., 410, 229 (1996).

6) E. Shouji, Y. Yokoyama, J. M. Pope, D. A. Buttry, and

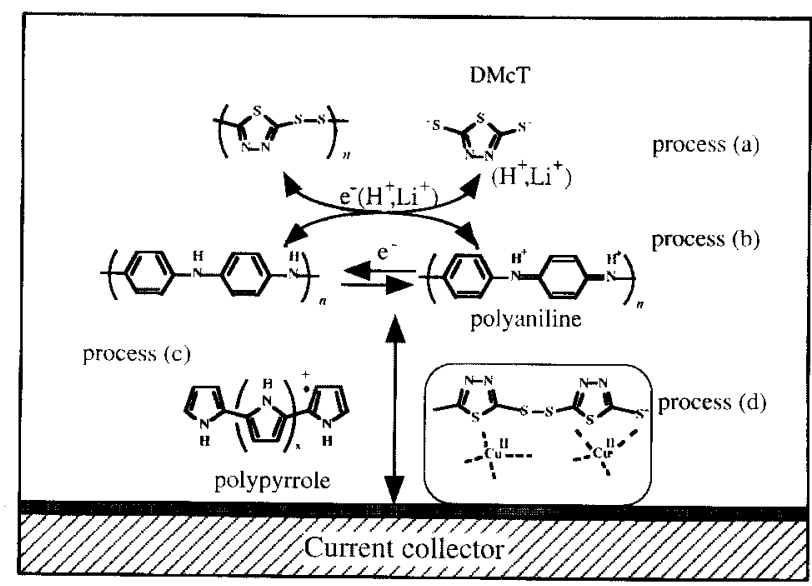

Scheme 2 Modeling of charge-transfer reactions in the composite cathode film on a copper current collector. 
N. Oyama, J. Phys. Chem.B, 101, 2861 (1997).

7) J. M. Pope and N. Oyama, J. Electrochem. Soc., 145, 1893 (1998).

8) J. M.Pope, T. Sato, E. Shouji, D. A. Buttry, T. Sotomura, and N. Oyama, J. Power Sources, 68, 739 (1997).

9) M. Liu, S. J. Visco, and L. C. De Jonghe, J. ElectroChem. Soc., 138, 1891 (1991).

10) M. M. Doeff, S. J. Visco, and L. C. De Jonghe, $J$. Electrochem. Soc., 139, 1808 (1992).

11) M. M. Doeff, S. J. Visco, and L. C. De Jonghe, $J$. Electrochem. Soc., 139, 2077 (1992).

12) M. Ue, S. J. Visco, and L. C. De Jonghe, Denki Kagaku(presently Electrochemistry), 61, 1409 (1993).

13) K. Daasbjerg, H. Jensen, R. Benassi, F. Taddei, S. Antonello, A. Gennaro, and F. Maran, J. Am. Chem. Soc., 121, 1750 (1999).

14) H. Daifuku, T. Kawagoe, and T. Matsunaga, Denki Kagaku(presently Electrochemistry), 57, 557 (1989).

15) For example, Y. Kojima, A. Utsuki, M. Kawasumi, A. Okada, Y. Fukushima, T. Kurauchi, and O. Kamigaito,
J. Mater. Res., 8, 1185 (1993).

16) T. Kobayashi and H.Yoneyama, J. Electroanal. Chem., 177, 293 (1984).

17) T. Sotomura, T. Tatsuma, and N. Oyama, J. Electrochem. Soc., 143, 3152 (1996).

18) N. Oyama, J. M. Pope, and T. Sotomura, J. Electrochem. Soc., 144, L47 (1997).

19) M. R. Gajendragad and U. Z. Agarwala, Anorg. Allg. Chem., 415, 84 (1975).

20) P. Ortega, L. R. Vera, and M. E. Guzman, Macromol. Chem. Phys., 198, 2949 (1997).

21) Q. Chi, T. Tatsuma, M. Ozaki, T. Sotomura, and N. Oyama, J. Electrochem. Soc., 145, 2369 (1998).

22) F. Matsumoto, M. Ozaki, S. C. Paulson, Y. Inatomi, and N. Oyama, Langmuir, 15, 857 (1999).

23) F. Matsumoto, Y. Inatomi, and N. Oyama, in preparation.

24) N. Oyama, S. Hirakawa, O. Hatozaki, and T. Sotomura, unpublished results.

25) T. Tatsuma, M. Taguchi, and N. Oyama, J. Electroanal. Chem., in press (1999). 